\title{
Pacific
}

Journal of

Mathematics

\section{AN ORTHOGONAL APPROACH TO THE SUBFACTOR OF A PLANAR ALGEBRA}

\author{
VAughan Jones, Dimitri Shlyakhtenko And KeVin WalKer
}




\title{
AN ORTHOGONAL APPROACH TO THE SUBFACTOR OF A PLANAR ALGEBRA
}

\author{
VAughan Jones, Dimitri Shlyakhtenko And KeVin Walker
}

By changing to an orthogonal basis, we give a short proof that the subfactor of the graded algebra of a planar algebra reproduces the planar algebra.

\section{Introduction}

Starting from a subfactor planar algebra, Guionnet, Jones and Shlyakhtenko [2007] constructed a tower of $\mathrm{II}_{1}$ factors whose standard invariant is precisely the given planar algebra. The construction was entirely in terms of planar diagrams and gave an alternate, diagrammatic proof of a result of Popa [1995]. The inspiration for the paper was from the theory of large random matrices, where expected values of words on random matrices give rise to a trace [Voiculescu 1985] on the algebra of noncommutative polynomials. Since that trace is definable entirely in terms of planar pictures, it was easy to generalize it to an arbitrary planar algebra, giving the planar algebra a concatenation multiplication to match that of noncommutative polynomials. Unfortunately, though the algebra structure is very straightforward, the inner product is not always easy to work with, as words of different lengths are not orthogonal. In this paper we use a simple diagrammatic orthogonalization discovered by $\mathrm{K}$. Walker, the third author, to prove again the $\mathrm{II}_{1}$ factor results of [Guionnet et al. 2007] in a direct and simple way without the use of full Fock spaces or graph $\mathrm{C}^{*}$-algebras. One may capitalize on the advantages of orthogonalization because the multiplication does not actually become much more complicated when transported to the orthogonal basis. We present the results by beginning with the orthogonal picture and giving a complete proof of the tower result. Then we show that this orthogonal structure is actually isomorphic to that of [Guionnet et al. 2007]. The same result was obtained simultaneously and independently by Kodiyalam and Sunder [2009].

MSC2000: 46L37.

Keywords: planar algebra, subfactor, free probability.

Jones was supported in part by NSF grant DMS-0401734 and DMS-0856316, Auckland University and the NZIMA. Shlyakhtenko was supported in part by NSF grants DMS-0555680 and DMS0900776. 


\section{Setup}

Let $\mathfrak{P}=\left(P_{n}\right)_{n=0,1,2, \ldots}$ be a subfactor planar algebra. Let $\mathrm{Gr}_{k}(\mathfrak{P})$ be the graded vector space $\bigoplus_{n \geq 0} P_{n+k}$ equipped with the pre-Hilbert space inner product $\langle\cdot, \cdot\rangle$, which makes it an orthogonal direct sum and for which

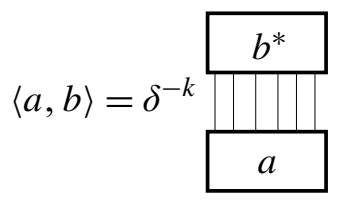

within $P_{n, k}$. Here we write $P_{n, k}$ for $P_{n+k}$ when it is considered as the $n$-graded part of $\mathrm{Gr}_{k}(\mathfrak{P})$. We will attempt to keep the pictures as uncluttered as possible by using several conventions and being as implicit as possible. Shadings, for instance, will always be implicit, and we will eliminate the outside boundary disc whenever convenient. An element $a \in P_{n, k}$ will be represented whenever possible in a picture as $-a$ - where the thick lines to the left and right of the box represent $k$ lines and the thick line at the top represents $2 n$ lines. If the multiple lines have to be divided into groups, the number of lines in each group will be indicated to the least extent necessary. The distinguished first interval in a box will always be the top left of the box. Thus the inner product above of $\langle a, b\rangle$ for elements of $P_{n, k}$ will be

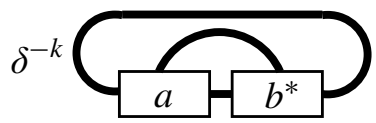

In the original works on planar algebras (for example, [Jones 1999]), each $P_{k}$ is an associative *-algebra whose product, with these conventions, views $P_{k}$ as $P_{0, k}$ and $a b$ as $a-b-$ By identifying $a$ with $a-a$, there are unital inclusions of $P_{k}$ in $P_{k+1}$. The identity element of $P_{k}$ is thus represented by a single thick horizontal line. It is also the identity element of $\mathrm{Gr}_{k}(\mathfrak{P})$. The trace $\operatorname{tr}$ (often called the Markov trace) on $P_{k}$ is normalized so as to be compatible with the inclusions by $\operatorname{tr}(a)=\delta^{-k}\langle a, 1\rangle$. We extend this trace to $\operatorname{Gr}_{k}(\mathfrak{P})$ by the same formula so that the trace of an element is the Markov trace of its zero-graded piece. Each $P_{n}$ is a finite-dimensional $\mathrm{C}^{*}$-algebra whose norms are also compatible with the inclusions.

\section{3. *-algebra structure on $\mathbf{G r}_{k}(\mathfrak{P})$}

Definition 3.1. If $a \in P_{m, k}$ and $b \in P_{n, k}$ are elements of $\mathrm{Gr}_{k}(\mathfrak{P})$, we define their product to be

$$
a \star b=\sum_{i=0}^{\min (2 m, 2 n)}
$$


where the $i$ means there are $i$ parallel strings. ${ }^{1}$ The numbers of other parallel strings are then implicitly defined by our conventions.

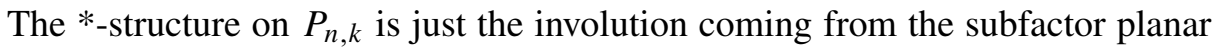
algebra.

Proposition 3.2. $\left(\mathrm{Gr}_{k}(\mathfrak{P}),{ }^{*}{ }^{*}\right)$ is an associative *algebra.

Proof. The property $(a \star b)^{*}=b^{*} \star a^{*}$ is immediate from the properties of a planar $*$-algebra. For associativity, note that both $a \star(b \star c)$ and $(a \star b) \star c$ are given by the sum over all epi (see Section 5) diagrams where no strand has both of its $a$, or both of its $b$, or both of its $c$. Here are two typical examples:
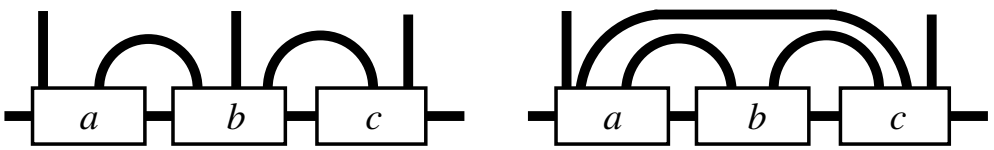

The inner product $\langle a, b\rangle$ is clearly equal to a positive multiple of $\operatorname{tr}\left(a b^{*}\right)$ and is positive definite by definition. We would like to perform the GNS construction, but since there is no $\mathrm{C}^{*}$-algebra available, we need to show by hand that left (and hence right) multiplication by elements of $\mathrm{Gr}_{k}(\mathfrak{P})$ is bounded.

Theorem 3.3. Let $a \in \mathrm{Gr}_{k}(\mathfrak{P})$. Then the map $L_{a}: \mathrm{Gr}_{k}(\mathfrak{P}) \rightarrow \mathrm{Gr}_{k}(\mathfrak{P}), \xi \mapsto=a \star \xi$, is bounded for the pre-Hilbert space structure.

Proof. We may suppose $a \in P_{n, k}$ for some $n$. Then $L_{a}$ is a sum of $2 n+1$ maps $L_{a}^{i}$ from an orthogonal direct sum of finite-dimensional Hilbert spaces to another, respecting the orthogonal decomposition, $L_{a}^{i}$ being the map defined by the $i$-th term in the sum defining $\star$. Thus it suffices to show that the norm of the map $L_{a}^{i}: P_{m, k} \rightarrow P_{m+n-i, k}$ is bounded independently of $m$, the number of $i$ values being at most $2 n+1$. Clearly we may suppose that $m \gg n+k$, which simplifies the number of pictures to be considered. So if $b \in P_{m, k}$, we must estimate $\langle a b, a b\rangle$, which is the following tangle:

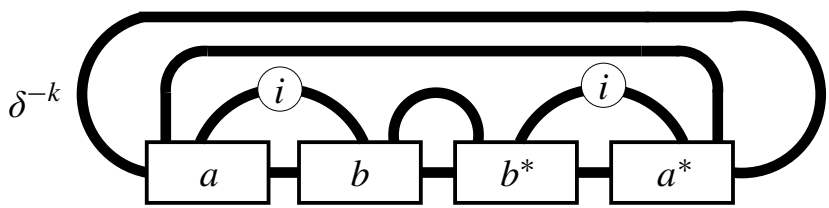

We may suppose $0 \leq i \leq 2 n$, since the norm of an operator is equal to that of its adjoint and the roles of $i$ and $2 n-i$ are reversed in going between $L_{a}^{i}$ and $\left(L_{a}^{i}\right)^{*}$. Then we may isotope the picture, putting $a$ and $b$ in boxes with the same number

${ }^{1}$ This algebra structure was pointed out to the first author by Roland Bacher in the mid 1990s. 
( $k+n$ and $k+m$ respectively) of boundary points on the top and bottom, and also possibly rotating them, to obtain the equivalent tangle

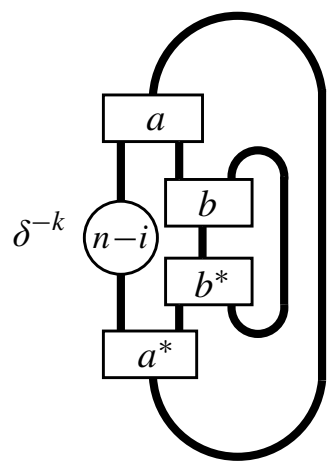

The multiplicities of all the strings are determined by the $n-i$ and our conventions.

Neglecting powers of $\delta$ that do not involve $m$, we see $\langle\tilde{a} \tilde{b}, \tilde{a} \tilde{b}\rangle$, where $\tilde{a}$ is $a$ with $m-i$ strings to the right, and $\tilde{b}$ is $b$ with $n-i$ strings to the left. The strings to the right do not change the norm of $a$ (as an element of the finite-dimensional $\mathrm{C}^{*}$-algebra $P_{k+n}$ ), by the uniqueness of the $\mathrm{C}^{*}$-norm. The $L^{2}$-norm of $\tilde{b}$ differs from that of $b$ by an $m$-independent power of $\delta$. Hence we are done.

$\mathrm{Gr}_{k}(\mathfrak{P})$ is thus what is sometimes called a Hilbert algebra or unitary algebra.

\section{The von Neumann algebras $M_{k}$.}

Definition 4.1. Let $M_{k}$ be the finite von Neumann algebra on the Hilbert space completion of $\mathrm{Gr}_{k}(\mathfrak{P})$ generated by left multiplication by the $L_{a}$.

Since right multiplication is also bounded, the identity in $P_{0, k}$ is a cyclic and separating trace vector for $M_{k}$ defining the faithful trace tr as usual, and the right multiplications generate the commutant of $M_{k}$. We shall first show that each $M_{k}$ is a factor.

Definition 4.2. The element $\cup_{k} \in P_{1, k}$ will be

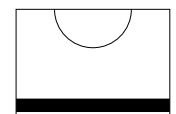
boundary to avoid disembodiment. The subalgebra of $\mathrm{Gr}_{k}(\mathfrak{P})$ generated by $\cup_{k}$ will be denoted $\mathfrak{A}_{k}$, and its weak closure in $M_{k}$ will be called $A_{k}$.

Definition 4.3. For $x \in P_{n, k}$ with $n \geq 0$ and $p, q \geq 0$, let $x_{p, q} \in P_{n+p+q, k}$ be

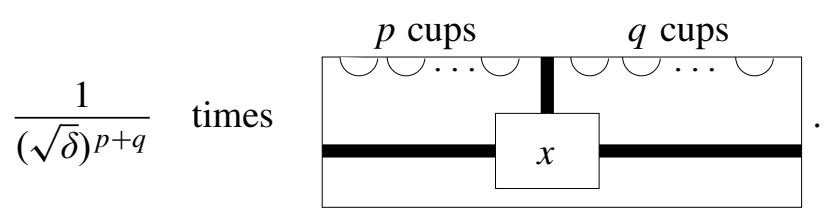


Definition 4.4. Let $W_{0}=\{0\} \subset P_{0, k}$, and for each $k \geq 1$, let $W_{n}$ be the span in $P_{n, k}$ of $\left\{x_{1,0}\right\}$ and $\left\{x_{0,1}\right\}$ for $x \in P_{n-1, k}$. For $n \geq 0$, let $V_{n}=W_{n}^{\perp}$.

Lemma 4.5. For $x \in P_{n, k}$, with $n \geq 1$,

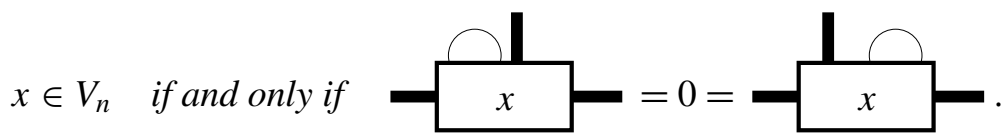

Proof. Taking the inner product of these two elements with an arbitrary element in $P_{n-1, k}$, we see the inner product of elements in $W_{n}$ with $x$.

Corollary 4.6. Let $v \in V_{m}$ with $m \geq 0$ and $v^{\prime} \in V_{n}$ with $n \geq 1$. Then

$$
\left\langle v_{p, q}, v_{p^{\prime}, q^{\prime}}^{\prime}\right\rangle= \begin{cases}\left\langle v, v^{\prime}\right\rangle & \text { if } p=p^{\prime} \text { and } q=q^{\prime}, \\ 0 & \text { otherwise. }\end{cases}
$$

Proof. If either $p \neq p^{\prime}$ or $q \neq q^{\prime}$, the left or rightmost pair of boundary points of $v$ or $v^{\prime}$ will be capped off to give zero.

Corollary 4.7. If $v \in V_{n}$ for $n>0$ is a unit vector, the $v_{p, q}$ are an orthonormal basis for the $\mathfrak{A}_{k}-\mathfrak{A}_{k}$ bimodule $\mathfrak{A}_{k} v \mathfrak{A}_{k}$.

Proof. By the previous lemma, it suffices to show that the span of the $v_{p, q}$ is invariant under left and right multiplication (using $\star$ ) by $\cup_{k}$. In fact, we have

$$
\cup_{k} \star v_{p, q}= \begin{cases}\sqrt{\delta} v_{1, q}+v_{0, q} & \text { if } p=0, \\ \sqrt{\delta} v_{p+1, q}+v_{p, q}+\sqrt{\delta} v_{p-1, q} & \text { otherwise; }\end{cases}
$$

and there is an obvious corresponding formula for right multiplication by $\cup_{k}$.

Lemma 4.8. The linear span of all the $v_{p, q}$ for $v \in V_{n}$ for all $n$ is $\mathrm{Gr}_{k}(\mathfrak{P})$.

Proof. By a simple induction on $n$ these vectors span $P_{n, k}$ for all $k$.

Let us summarize all we have learned using the unilateral shift $S$ (with $S^{*} S=1$ ) on $\ell^{2}(\mathbb{N})$.

Theorem 4.9. Suppose $\delta>1$. As an $A_{k}-A_{k}$ bimodule,

$$
L^{2}\left(M_{k}\right)=P_{0, k} \otimes \ell^{2}(\mathbb{N}) \oplus\left\{\mathfrak{H} \otimes \ell^{2}(\mathbb{N}) \otimes \ell^{2}(\mathbb{N})\right\},
$$

with $\cup_{k}$ acting on the left and right on $P_{0, k} \otimes \ell^{2}(\mathbb{N})$ by $\mathrm{id} \otimes\left(\sqrt{\delta}\left(S+S^{*}\right)+S S^{*}\right)$, on the left on $\mathfrak{H} \otimes \ell^{2}(\mathbb{N}) \otimes \ell^{2}(\mathbb{N})$ by id $\otimes\left(\sqrt{\delta}\left(S+S^{*}\right)+1\right) \otimes \mathrm{id}$, and on the right on $\mathfrak{H} \otimes \ell^{2}(\mathbb{N}) \otimes \ell^{2}(\mathbb{N})$ by $\mathrm{id} \otimes \mathrm{id} \otimes\left(\sqrt{\delta}\left(S+S^{*}\right)+1\right)$. (Here $\mathfrak{H}$ is an auxiliary infinite-dimensional Hilbert space.)

Proof. Obviously $P_{0, k}$ commutes with $A_{k}$, so the first term in the direct sum is the result of a simple calculation. Choosing an orthonormal basis for each $V_{n}$ gives the rest by Corollary 4.7 .

Corollary 4.10. $A_{k}^{\prime} \cap M_{k}=A_{k} P_{0, k}$. 
Proof. It suffices to show that no nonzero $\xi \in \ell^{2}(\mathbb{N}) \otimes \ell^{2}(\mathbb{N})$ satisfies $\left(S+S^{*}\right) \xi=$ $\xi\left(S+S^{*}\right)$. But such a $\xi$ would be a Hilbert-Schmidt operator on $\ell^{2}(\mathbb{N})$ commuting with $S+S^{*}$, and $S+S^{*}$ would leave invariant a finite-dimensional subspace and hence have an eigenvalue. But $S+S^{*}$ is Voiculescu's semicircular element and is known not to have an eigenvalue (this follows immediately from a direct proof).

Corollary 4.11. Suppose $\delta>1$. For each $k, M_{0}^{\prime} \cap M_{k}=P_{0, k}$ (as an algebra).

Proof. The element $\alpha=\square$ is in $M_{0} \subset M_{k}$, so it is enough to show that the only elements in the Hilbert space closure of $P_{0, k} A_{k}$ that commute with it are elements of $P_{0, k}$. We define

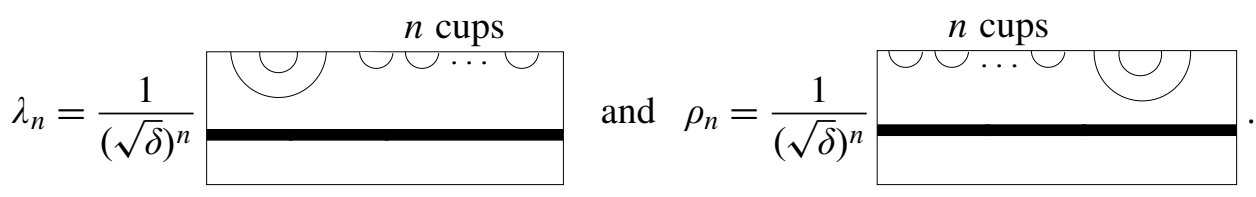

We have

$$
\left[\alpha, 1_{0, n}\right]=\left(\lambda_{n}-\rho_{n}\right)+\frac{1}{\sqrt{\delta}}\left(\lambda_{n-1}-\rho_{n-1}\right) .
$$

So if an element $c$ in the closure of $P_{0, k} A_{k}$ is written as an $\ell^{2} \operatorname{sum} \sum_{n=0}^{\infty} c_{n} \star 1_{0, n}$ with $c_{n} \in P_{0, k}$, we find

$$
[\alpha, c]=\sum_{n=1}^{\infty}\left(c_{n}+\frac{1}{\sqrt{\delta}} c_{n+1}\right) \star\left(\lambda_{n}-\rho_{n}\right) .
$$

The terms in the sum are orthogonal for different $n$, so for $c$ to commute with $\alpha$, we would have $c_{n+1}=-\delta c_{n}$ for $n>1$, which forces $c_{n}=0$ for $n \geq 1$ since $c_{n} \in \ell^{2}$. So $c \in P_{0, k}$.

Corollary 4.12. If $\delta>1, M_{k}$ is a type $\mathrm{II}_{1}$ factor.

Proof. If $x$ were in the center of $M_{k}$, it would have to be in $P_{0, k}$. But a trivial diagrammatic argument shows that the only elements in $P_{0, k}$ that commute with $\checkmark$ are scalar multiples of the identity.

We now want to identify the $\mathrm{II}_{1}$ factors $M_{k}$ with the tower coming from the subfactor $M_{0} \subset M_{1}$ obtained by iterating the basic construction of [Jones 1983]. For simplicity we will do it for the case $M_{0}, M_{1}, M_{2}$, with the general case following the same argument but with heavier notation.

Definition 4.13. The element $e \in M_{2}$ will be $(1 / \delta)$

Proposition 4.14. This $e$ is a projection, and exe $=E_{M_{0}}(x) e$ for $x \in M_{1}$, and $E_{M_{1}}(e)=\delta^{-2} \mathrm{id}$, with $E_{M_{i}}$ the trace-preserving conditional expectation onto $M_{i}$.

Proof. An easy computation with diagrams. 
Lemma 4.15. The von Neumann algebra $\left\{M_{1}, e\right\}^{\prime \prime}$ is a $\mathrm{II}_{1}$ factor.

Proof. If $x$ is in the center of $\left\{M_{1}, e\right\}^{\prime \prime}$, then it commutes with $M_{0}$, so by 4.11 we know that $x \in P_{0,2}$. But $x$ also has to commute with $\square$, which forces $x$ to be of the form

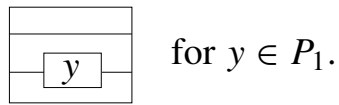

But for this to commute with $e$, it must be a scalar multiple of the identity.

Corollary 4.16. For $z \in\left\{M_{1}, e\right\}^{\prime \prime}$, we have $z e=\delta^{2} E_{M_{1}}(z e) e$.

Proof. By algebra, $M$ and $M e M$ span a *-subalgebra of $\left\{M_{1}, e\right\}^{\prime \prime}$, which is thus weakly dense. The assertion is trivial for $z \in M$ and a simple calculation for $z \in M_{1} e M_{1}$. Also $E_{M_{1}}$ is continuous.

Corollary 4.17. The map $x \mapsto \delta x$ from $M_{1}$ to $\left\{M_{1}, e\right\}^{\prime \prime} e$ is a surjective isometry intertwining $E_{M_{0}}$ on $L^{2}\left(M_{1}\right)$ and left multiplication by .

Proof. Surjectivity follows from the previous lemma. The intertwining property is a calculation.

Corollary 4.18. $\left[M_{1}: M_{0}\right]=\delta^{2}$. The basic construction for $M_{0} \subset M_{1}$ is $\left\{M_{1}, e\right\}^{\prime \prime}$.

Proof. The basic construction is the von Neumann algebra on $L^{2}\left(M_{1}\right)$ generated by $E_{M_{0}}$ and $M_{1}$. By Lemma $4.15,\left\{M_{1}, e\right\}^{\prime \prime}$ as a subalgebra of $M_{2}$ is the same, as it is acting on $\left\{M_{1}, e\right\}^{\prime \prime} e$ by left multiplication. Also this is the basic construction by the previous corollary. The index is then just a matter of evaluating the trace of $e$, by uniqueness of the trace on a factor.

Corollary 4.19. $\left\{M_{1}, e\right\}^{\prime \prime}=M_{2}$.

Proof. The same argument as above applied to $M_{1} \subset M_{2}$ shows that $\left[M_{2}: M_{1}\right]=\delta^{2}$. But then $\left[M_{2}:\left\{M_{1}, e\right\}^{\prime \prime}\right]=1$.

Summing up the above arguments applied to the whole tower, we have:

Theorem 4.20. Let $\mathfrak{M}_{n}$ be the $\mathrm{II}_{1}$ factor obtained by the basic construction from $\mathfrak{M}_{n-2} \subset \mathfrak{M}_{n-1}$ with $\mathfrak{M}_{0}=M_{0}$ and $\mathfrak{M}_{1}=M_{1}$, and suppose $e_{n}$ is the projection of the basic construction generating $\mathfrak{M}_{n+1}$ from $\mathfrak{M}_{n}$. Then there is a (unique) isomorphism of towers from $\mathfrak{M}_{n}$ to $M_{n}$ that is the identity on $M_{1}$ and sends $e_{i}$ to

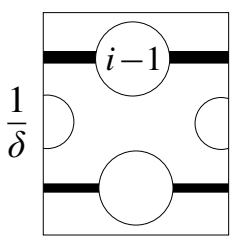

Theorem 4.21. Given a subfactor planar algebra $\mathfrak{P}=\left(P_{n}\right)$ with $\delta>1$, the subfactor $M_{0}$ constructed above has planar algebra invariant equal to $\mathfrak{P}$. 
Proof. It is well known [Jones 1999] that the planar algebra structure is determined by knowledge of the $e_{i}$, the multiplication, and the embeddings $P_{n} \subset P_{n+1}$ corresponding to the inclusions $M_{1}^{\prime} \cap M_{n+1} \subset M_{0}^{\prime} \cap M_{n+1}$ and $M_{0}^{\prime} \cap M_{n} \subset M_{0}^{\prime} \cap M_{n+1}$. The conditional expectations onto these are just given by the appropriate diagrams.

\section{Change of basis}

In this section we show that the pre-Hilbert space $\mathrm{Gr}_{k}(\mathfrak{P})$ defined above is isometric and isomorphic as a *-algebra to the pre-Hilbert space also called $\mathrm{Gr}_{k}(\mathfrak{P})$ defined in [Guionnet et al. 2007]. To distinguish between them, we will call the latter pre-Hilbert space $\operatorname{Hr}_{k}(\mathfrak{P})$.

Recall that $\operatorname{Hr}_{k}(\mathfrak{P})$ is defined on the same underlying vector space $\bigoplus_{n \geq 0} P_{n+k}$, but with a simpler multiplication and more complicated inner product. The multiplication is the simple juxtaposition

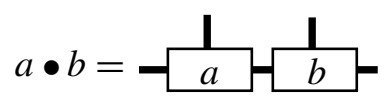

while the inner product $\langle\langle a, b\rangle\rangle$ of $a \in P_{m, k}$ and $b \in P_{n, k}$ is

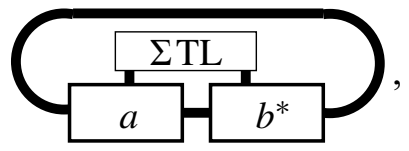

where $\Sigma \mathrm{TL}$ is the sum of all loopless Temperley-Lieb diagrams with $2(m+n)$ strands on the boundary. Note that while the multiplication respects the grading, the inner product does not.

We will define an upper-triangular change of basis in $\bigoplus_{n \geq 0} P_{n+k}$, which induces an isomorphism between $\mathrm{Gr}_{k}(\mathfrak{P})$ and $\mathrm{Hr}_{k}(\mathfrak{P})$.

Recall that an epi TL diagram is one in which each point on the top/outgoing side of the rectangle is connected to the bottom/incoming side of the rectangle. A monic diagram is defined similarly, but with the roles of the sides reversed; see Figure 1.

Note that each TL diagram factors uniquely as epi followed by monic.

We will think of a TL diagram with $2 i$ strands on the bottom of the rectangle and $2 j$ strands on the top of the rectangle as a linear map from $P_{i, k}$ to $P_{j, k}$ (here $k$ is arbitrary and fixed).

We define

$$
X: \bigoplus_{n \geq 0} P_{n+k} \rightarrow \bigoplus_{n \geq 0} P_{n+k}
$$



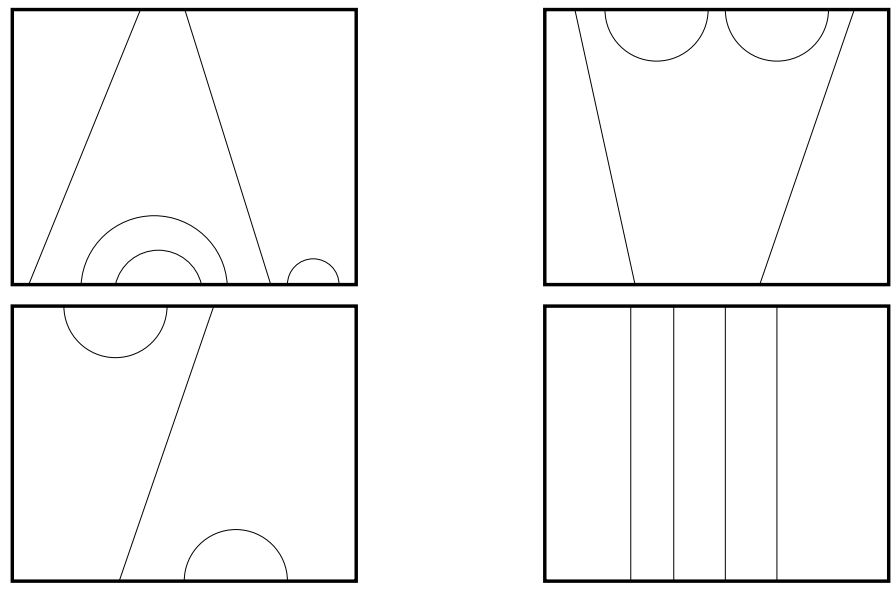

Figure 1. From upper left, clockwise: epi, monic, both epi and monic, neither epi nor monic.

to be the sum of all epi TL diagrams. Thus the $j, i$ block of $X$ is the (finite) sum of all epi TL diagrams from $2 i$ strands to $2 j$ strands, which is the identity if $i=j$ and zero if $i<j$.

We define a nonnested epi TL diagram to be one where each "turn-back" or "cap" on the bottom of the rectangle encloses no other turn-backs. We define

$$
Y: \bigoplus_{n \geq 0} P_{n+k} \rightarrow \bigoplus_{n \geq 0} P_{n+k}
$$

to be the sum of all nonnested epi TL diagrams, with the coefficient in the $i, j$ block equal to $(-1)^{i-j}$.

Remark 5.0.1. In the special case of a vertex model planar algebra [Jones 1999], the graded vector space is the (even degree) noncommutative polynomials. In [Voiculescu 1985], a map was defined from these polynomials to full Fock space, the vacuum component of which is the trace on what we have called $\operatorname{Hr}_{k}(\mathfrak{P})$. In this case the map $X$ gives Voiculescu's map in its entirety and $Y$ is its inverse. We presume that these formulas are known perhaps in some slightly different form, but have been unable to find them explicitly in the literature.

Lemma 5.1. $X Y=1=Y X$.

Proof. $X_{j m} Y_{m i}$ is equal to the sum of all products of a nonnested TL diagram from $i$ to $m$ (with $i-m$ turn-backs) followed by a general epi TL diagram from $m$ to $j$, with sign $(-1)^{i-m}$. The number of times a given diagram $D$ appears in this sum is equal to the number of subsets of size $i-m$ taken from the innermost turn-backs of $D$. It follows that the total coefficient of $D$ in $\sum_{m} X_{j m} Y_{m i}$ is $\sum_{p}(-1)^{p}\left(\begin{array}{l}t \\ p\end{array}\right)=0$ 
(assuming $p>0$ ), where $t$ is the total number of innermost turn-backs of $D$. Thus the off-diagonal blocks of $X Y$ are zero, and it is easy to see that the diagonal blocks of $X Y$ are all the identity.

The proof that $Y X=1$ is similar, with outermost turn-backs playing the role previously played by innermost turn-backs.

\section{Lemma 5.2. $X(a \bullet b)=X(a) \star X(b)$.}

Proof. Let $a \in P_{m, k}$ and $b \in P_{n, k}$. Each epi diagram from $2(m+n)$ to $2 j$ appearing in the definition of $X(a \bullet b)$ factors uniquely as $T \cdot(L \mid R)$, where $L$ is an epi diagram from $2 m$ to $2 m^{\prime}, R$ is an epi diagram from $2 n$ to $2 n^{\prime}, L \mid R$ denotes $L$ and $R$ placed side by side, and $T$ is an epi diagram from $2\left(m^{\prime}+n^{\prime}\right)$ to $2 j$ where each turn-back has one end in the $m^{\prime}$ side and the other end in the $n^{\prime}$ side. $L$ corresponds to a diagram used in the definition of $X(a), R$ corresponds to a diagram used in the definition of $X(b)$, and $T$ corresponds to a diagram used in the definition of $\star$ in $X(a) \star X(b)$.

Lemma 5.3. $\langle\langle a, b\rangle\rangle=\langle X(a), X(b)\rangle$.

Proof. Let $a \in P_{m, k}$ and $b \in P_{n, k}$. Let $D$ be a TL diagram in $\Sigma$ TL used in the definition of $\langle\langle a, b\rangle\rangle$. We can think of $D$ as a TL diagram from $2 m$ strands to $2 n$ strands, and from this point of view it has a unique factorization $E \cdot M$, where $E$ is an epi diagram starting at $2 m$ and $M$ is a monic diagram ending at $2 n$. $E$ is an epi diagram figuring in the definition of $X(a)$, and $M^{*}$ is an epi diagram figuring in the definition of $X(b)$. The way in which $E$ and $M^{*}$ are glued together corresponds to the definition of $\langle\langle\cdot, \cdot\rangle\rangle$.

\section{References}

[Guionnet et al. 2007] A. Guionnet, V. F. R. Jones, and D. Shlyakhtenko, "Random matrices, free probability, planar algebras and subfactors", preprint, 2007. arXiv 0712.2904

[Jones 1983] V. F. R. Jones, "Index for subfactors”, Invent. Math. 72:1 (1983), 1-25. MR 84d:46097 Zbl 0508.46040

[Jones 1999] V. Jones, "Planar algebras", preprint, University of California, Berkeley, 1999, available at http://www.math.berkeley.edu/ vfr/plnalg1.ps.

[Kodiyalam and Sunder 2009] V. Kodiyalam and V. S. Sunder, "From subfactor planar algebras to subfactors”, Internat. J. Math. 20:10 (2009), 1207-1231. MR 2574313 Zbl 05638350

[Popa 1995] S. Popa, "An axiomatization of the lattice of higher relative commutants of a subfactor", Invent. Math. 120:3 (1995), 427-445. MR 96g:46051 Zbl 0831.46069

[Voiculescu 1985] D. Voiculescu, "Symmetries of some reduced free product $C^{*}$-algebras", pp. 556-588 in Operator algebras and their connections with topology and ergodic theory (Buşteni, 1983), edited by H. Araki et al., Lecture Notes in Math. 1132, Springer, Berlin, 1985. MR 87d: $46075 \mathrm{Zbl} 0618.46048$

Received July 1, 2009. 
VAUGHAN JONES

DEPARTMENT OF MATHEMATICS

UNIVERSITY OF CALIFORNIA

BERKELEY, CA 94720

UNITED STATES

vfr@math.berkeley.edu

http://math.berkeley.edu/ vfr/

DIMITRI SHLYAKHTENKO

DEPARTMENT OF MATHEMATICS

UNIVERSITY OF CALIFORNIA

Los ANGeles, CA 90095

UNITED STATES

shlyakht@math.ucla.edu

http://www.math.ucla.edu/ shlyakht/

KeVIn WALKer

MicRosofT STATION Q

UNIVERSITY OF CALIFORNIA

SANTA BARBARA, CA 93106

UNITED STATES

kevin@canyon23.net

http://canyon23.net/math/ 